pregnancy and 9 in post-partum. The frequency of NL was estimated at 9.8\% [95\% CI 6.3-14.9] $\quad(\mathrm{CHB}=6.2 \% \quad[\mathrm{IC} 95 \%$ $3.5-10.7])$. Table 1 describes the maternal and fetal characteristics of NL cases. The most frequent manifestations were skin $(n=7)$ and cardiac involvement $(n=12)$. In 1 case, there was a history of NL in a previous pregnancy. Of the patients with $\mathrm{CHB}, 5$ required a pacemaker.

Conclusions In conclusion, the frequency of NL in our multicentric cohort is greater than other international cohorts. Differences could be related to genetic/environmental factors as well as methodological limitations and selection bias.

Funding Source(s): None

\section{FLUCTUATION, INVISIBILITY, FATIGUE THE BARRIERS TO MAINTAINING EMPLOYMENT IN SLE: RESULTS OF AN ON-LINE SURVEY}

${ }^{1}$ Sara Booth*, ${ }^{2}$ Elizabeth Walker, ${ }^{2}$ Elizabeth Price. ${ }^{1}$ University of Cambridge; ${ }^{2}$ Faculty of Health Sciences Hull University

10.1136/lupus-2019-Ism.272

Background It is well-documented that systemic lupus erythematosus (SLE) is associated with high levels of workplace disability and unemployment. The objective of this study was to understand reasons for this and to describe the barriers and facilitators to employment identified by people with SLE to develop appropriate solutions. Unemployment, as well as unsuitable work, has adverse health outcomes.

Methods Adults with SLE completed a UK-specific online survey, through the LUPUS UK website, designed to find out more about the difficulties and successes that people with SLE have in maintaining employment. The survey was predominantly qualitative, to understand participants employment experiences to generate possible solutions.

Results 393 people gave detailed responses to the survey within eight weeks. Every respondent reported a detrimental effect of SLE on their ability to work. $40.45 \%$ had left employment because of it. The themes of concern to respondents were unambiguous (i) the difficulties of working (and career damage) with SLE (ii) long-term fear and anxiety overshadowing work/family life (iii) the greater potential to remain in some/employment when modifications of work pattern and support from management and colleagues were available. Antidisability discrimination was reported as partially helpful but incomplete, omitting many features of lupus disability. SLErelated fatigue, its invisibility and fluctuating nature were felt to be the main barriers to maintaining employment. Many respondents could work only part time, anxiety was high regarding future ability to continue working and financial strains. Many had taken substantial pay reductions and refused offered promotions to preserve their health. Distress due to loss of work and the benefits it brings were reported by every respondent who had left work. Loss of work and the demeaning impact of a widespread lack of understanding of the reasons for workplace disability had a detrimental effect on individuals mental health.

Conclusions SLE presents specific difficulties for maintaining employment fatigue, fluctuation and invisibility not addressed by current anti-discrimination legislation or currently-available reasonable adjustments. This study demonstrates that (i) employment is an important area of concern for people with SLE (ii) SLE has significant detrimental effects on individuals' ability to participate and progress in employment (iii) legislators and employers need information about SLE as invisibility and fluctuation cause hidden problems (ii) more data is needed to inform workplace adjustments if individual distress and societal loss of skills are to be addressed.

Funding Source(s): No external funding required

\section{SAFETY OF RESEARCH CORES OBTAINED FROM CLINICALLY INDICATED BIOPSIES IN THE ACCELERATING MEDICINES PARTNERSHIP NETWORK}

${ }^{1}$ Jill Buyon*, 'Michael Belmont, ${ }^{1}$ Peter Izmirly, ${ }^{1}$ Nicole Bornkamp, ${ }^{1}$ Catherine Trad, ${ }^{1}$ Rohit Bhan, ${ }^{1}$ Kimberly Robins, ${ }^{1}$ Robert Clancy, ${ }^{2}$ Chaim Putterman, ${ }^{1}$ Ming Wu, AMP Network. 'NYU School of Medicine; ${ }^{2}$ Albert Einstein College of Medicine and Montefiore Medical Center

\subsection{6/lupus-2019-Ism.273}

Background The Accelerating Medicines Partnership (AMP) is a public-private network comprising the National Institutes of Health, medical centers, biopharmaceutical companies and non-profit organizations. The Networks overarching mission is to develop SLE diagnostics and treatments via transcriptomic analysis of cells isolated from renal biopsies. This study addresses the safety of the research component of clinically indicated percutaneous kidney biopsies in anticipation of generalizing these results for real world clinical practice. We focused on one site (NYU METRO) for uniformity of data collection in which all biopsies were done by interventional radiology using an 18 gauge needle.

Methods Patients undergoing kidney biopsies to evaluate proteinuria exceeding $500 \mathrm{mg} / \mathrm{day}$ (based on uPCR) were consented to donate research tissue which was acquired via an extra pass or the use of a piece of a core with sufficient glomeruli available for both clinical evaluation and research. Adverse events (AE) within 30 days of biopsy were reported.

Results 98 patients with sufficient research kidney tissue have enrolled through the three Phases of AMP $(0=$ technical, $1=$ technical translational, $2=$ ongoing full follow-up): 10 males, 88 females; 68 Non-Hispanic (69.4\%), 30 Hispanic (30.6\%); 19 Asian (19.4\%), 32 Black (32.7\%), 46 White (46.9\%), 1 other (1.0\%). Biopsy classes varied: 5 non-LN (5.1\%), 1 Class I (1.0\%), 5 Class II (5.1\%), 21 Class III (21.4\%), 16 Class IV (16.3\%), 15 Class V (15.3\%), 20 Class III/V (20.4\%), 11 Class IV/V (11.2\%), 3 Class VI (3.1\%), 1 not yet determined. Research biopsy core lengths ranged from 4-19 mm. Eleven patients had an AE (research biopsy core lengths $513 \mathrm{~mm}$ ); 8 were hematomas (only 6 had an extra pass specific for research). Of the 8 patients, 5 were hospitalized, 4 for the hematoma with 1 receiving a transfusion. Neither number of passes nor research core length predicted hematoma. All hematomas resolved within one week. The remaining 3 AEs were unrelated to the biopsy: 1 cardiac event-related death, 1 UTI, 1 fall. This site-specific frequency is similar to that being observed in the overall multisite ongoing Phase 2 AMP Network.

Conclusions Albeit this detailed report is limited to one site, it is consistent with the Network experience. These data support the feasibility and safety of obtaining a research core to bring personalized medicine to the management of LN.

Funding Source(s): This work was supported by the Accelerating Medicines Partnership (AMP) in Rheumatoid Arthritis and Lupus Network. AMP is a public-private partnership (AbbVie, Arthritis Foundation, Bristol-Myers Squibb, Foundation for the 